\title{
Methods of Modeling the Process of Building the Conceptual Model of TIAV Multimedia System
}

\author{
Beknazarova Saida Safibullayevna \\ Tashkent University of Information Technologies, Uzbekistan
}

Copyright $@ 2015$ Horizon Research Publishing All rights reserved.

\begin{abstract}
In the article describes methods for modeling the process of building a conceptual model of a multimedia system, described the principal model multimedia system, the development of an algorithm for constructing online system - Designer TIAV-multimedia systems are described information-functional model of the online system constructor TIAV-multimedia systems, presents the conceptual model of the process processing of information resources in TIAV-multimedia systems, as well as the information considered simulation model of discrete-continuous processes processing of information resources.
\end{abstract}

Keywords Conceptual Model, Multimedia System, Designer TIAV-Multimedia Systems, Constructor TIAV-Multimedia Systems

\section{Introduction}

Multimedia systems are a necessary part of the process of using information resources of society, and the pace of their development is determined to a large extent the rate of accumulation of professional knowledge.

Acceleration of the dynamics of processes in all spheres of human activity, the complexity of industrial production, social, economic and political life naturally led, on the one hand, a rapid increase in demand for information knowledge, and on the other - to the creation of new tools and technologies to meet these needs. Intensive development of scientific and technological progress and the emergence of modern computer and telecommunication technologies and equipment, capable of storing, processing, and provide various types of discrete-continuous media, the emergence and development of multimedia systems and related methodological innovations radically changed the approach to the implementation of educational activities, to intensify the process of preparation professionals at all levels of the education system. Scope of multimedia information systems expands with each passing day. Today, they cover a wide range of applications including classrooms, conference rooms, comprehensive monitoring systems, situational centers and control centers, etc. Development of multimedia systems is characterized by the increasing complexity of their architecture. All processes in multimedia systems are discrete-continuous, as a result, there is a need to develop models of efficient algorithms, software package such automated multimedia systems, which would include online designers design and create multimedia courseware, training multi contents that based on the use of discrete-continuous object class TIAV.

TIAV- multimedia system - a class of objects consisting of textual, illustrative, video and audio information streams that form the basis for the development of multimedia content.

A retrospective analysis of the theory, problems and challenges of designing multimedia system shows the difficulties for the creation of information, functional, dynamic models of efficient algorithms and implementation of the organizational structure of software systems to meet the challenges of structuring discrete continuous flow of information resources. Thus, the construction of mathematical and information models and efficient algorithms based on them develop problem-oriented software systems for the design of control systems for discrete-continuous processes processing of information resources in TIAV-media systems is an urgent task.

\section{Materials and Methods}

Model of the control system TIAV (text, image, audio, video) - discrete-continuous processes in a multimedia system, which operates under base-loaded available external subsystems trainees, such as the level of creating multi contents level passing the examination, the level of multi contents study, the level of control of knowledge; level of publication, broadcast multimedia resources in the pages of the system. Model of the control system TIAVdiscrete-continuous process of multimedia system is shown in Fig 1. 


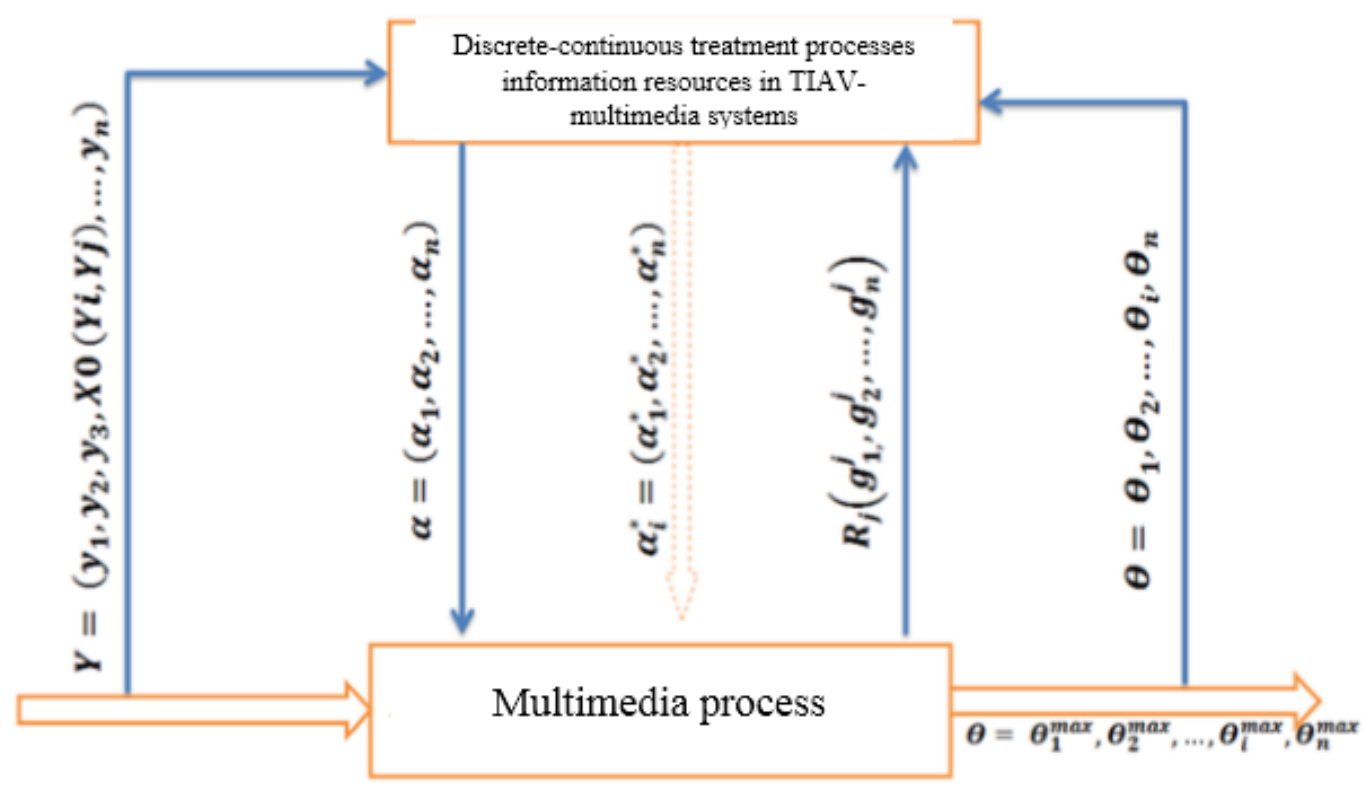

Figure 1. Model of the control system TIAV- discrete-continuous process in multimedia system

where $y_{0}, y_{1}, y_{2}, y_{3}$, the initial characteristics of excellence with the listener, $y_{0}$ - the availability of knowledge on text, $y_{1}$ -the availability of knowledge on graphics, illustrations, $y_{2}$-the availability of knowledge for the study of video, $y_{3^{-}}$ the knowledge of animation or simulation models, $X_{0}\left(y_{i}, y_{j}\right)$ - the functional integrity of the $y_{i}=<M_{i}, N_{i}>$, predicate, which $M_{i}$ reflects the legitimacy of the transition between them. At each stage of the life cycle multi content, parameters where the parameter is supplemented by the addition of quality indicators and $\mathrm{k}$ sets of control points $\mathrm{z}$; $\alpha=\left(\alpha_{1}, \alpha_{2}, \ldots, \alpha_{n}\right)$-vector of priorities of some criteria over others: to summarize the elements of each row and each normalized by dividing the sum of the sum of all the elements; the sum of the results obtained will be equal to one; $R_{j}\left(g_{1,}^{j}, g_{2}^{j}, \ldots, g_{n}^{j}\right)$ - effective solutions, $R_{j}$ - objects multi objective tasks for which each criterion is satisfied constraint type, $g_{i}^{j} \geq F_{i}$ where $\mathrm{i}=1,2, \ldots, F_{i}$, - the maximum permissible values of selected consumer quality criteria; $\alpha_{i}^{*}=\left(\alpha_{1}^{*}, \alpha_{2}^{*}, \ldots, \alpha_{n}^{*}\right)$ - the parameters of a single indicator the quality of the developed of multi content. This modification will be a compromise approach that allows to obtain acceptable results in terms of accuracy at low cost time; $\theta=\theta_{1}, \theta_{2}, \ldots, \theta_{i}, \theta_{n}$ - for each of the criteria selected among intermediate values of quality criteria discussed, results in a vector indicating the desired optimum $(\theta)$, which is brought to a maximum value; $\theta^{\max }=$ $\theta_{1}^{\max }, \theta_{2}^{\max }, \ldots, \theta_{i}^{\max }, \theta_{n}^{\max }$-by each criterion selected maximum value among the quality criteria discussed, results in a vector indicating the desired optimum $\left(\theta^{\max }\right)$.

Let multimedia system consists of $k_{n}$ multi contents. To improve media gram level test, the system should provide the optimum level of knowledge for the chosen specialty, which tends to the maximum value $\theta \rightarrow \max$. To do this, the software that allows you to generate a class for multi contents disciplines specialties, created on the basis of algorithms to identify the parameters of the distribution in terms of the objective $a_{11} k_{1}, a_{12} k_{2}, \ldots, a_{i j} k_{n}$ multi contents directory. The analysis is based on a probabilistic model search. In each class of multi contents proposed system formed of multi contents.

Multi contents considered valid class is a class in which the requirements are met:

$$
\left\{\begin{array}{c}
\sum_{i=1}^{N} k_{i} \geq M \\
k_{e l} \geq M_{e n} \\
M_{u m i n} \leq \sum_{i=1}^{N} k_{i} \leq M_{u \max }, \forall_{i} \in U \\
\sum_{i=1}^{N} k_{i j} \geq M_{\min } \\
\sum_{j=1}^{R} d_{i j}>0, \forall_{i} \in D_{A \cup b} \\
\sum_{j=1}^{R} d_{i j} \leq 1, \forall_{i} \\
\forall_{i} \sum_{i=1}^{N} \sum_{k=1}^{R} a_{i l} \cdot d_{l k} \cdot p_{j k}=\delta \sum_{i=1}^{N} a_{i l} \\
\forall_{i} \sum_{i=1}^{N} \sum_{k=1}^{R} a_{i l} d_{l k}<\lambda \sum_{i=1}^{N} a_{i l}(k-j-1)
\end{array}\right\}
$$

Where $k_{i}$ - integer lessons multi content $i$,

$\mathrm{M}$ - the minimum number of lessons theoretical training, $M_{\text {umin }}-M_{\text {umax }}$ - number of lessons of multi contents,

$p_{j k}=\left\{\begin{array}{l}1, \text { if } k<j \\ 0, \text { if } k \geq j\end{array}\right\}$, there is a variable, the semantics of which suggests the possibility of studying in class 1 multi content multi contents $\mathrm{j}$ only when the preceding multi content studied in earlier studies; $\delta=0,1$ - coefficient of possible error, $a_{i j}$ - coefficients closeness of the connection between of multi content $i$ - share multi content needed to learn multi 
content $\mathrm{j}$, take values $a_{i j}$ in the range $[0,1]$. The values of coefficients closeness of the connection between of multi contents $i$ established on the basis of expert estimates, based on the assumption that the true value of the characteristic is within the range of estimates of experts and generalized collective opinion is valid. Values of the coefficients of competence allow us to simplify the procedure for determining the consistency of their views and suppress the possibility of filling the system unreliable knowledge. There is a need to address a number of problems:

1.Formirovat multi contents class, based on multi criteria problem, the importance of maximizing the total for training multi contents in obtaining optimal knowledge in the chosen direction, specialization $S_{p k}$, suggesting selection for each step multi contents with the highest rate of professional relevance;

2. Determine the set of multi contents multimedia work, in which form separate competencies that define the subject area of competence formation of private training.

1. Formal job quality criterion has the form:

$$
Q_{1}: \sum_{i=1}^{N} \sum_{j=1}^{R} W_{i k} d_{i j} \rightarrow \max
$$

where $W_{j k}$ - the coefficient of professional importance (weight) media course i specialization $S_{p k}, d_{i j}$ - parameter defining the study multi content $i$ in class multi contents.

Set of feasible solutions is multi contents class that can be assigned from the current state of the system $S_{h}$, this class $X_{h}$ consists of multi-course, no ancestors or all ancestors are studied, which in this case fit the constraints. That is, based on the information on the results of the study multi contents can predict class multi contents, the study of which is possible with the next study multi contents.

2. The problem of estimating the level of formation of competence is to calculate the student with known values of the variables $x_{r}^{t}, \delta_{i j k}^{t}, z_{r i j k}^{t}$, the value assessment $y_{0}^{t}=$ $F\left(x_{r}^{t}, \delta_{i j k}^{t}, z_{r i j k}^{t}\right)$. The result set of multimedia courseware development class trained in time $\mathrm{t}$ is denoted $X_{\text {ОКМ }}^{t}=$ $\left(x_{1}^{t}, \ldots, x_{m^{t}}^{t}\right)$. The result set TIAV multimedia performance listener in time $\mathrm{t}$ is denoted $X_{\mathrm{MOP}}^{t}=\left(x_{m^{t}+1}^{t}, \ldots, x_{m^{t}+p^{t}}^{t}\right)$, while $-p^{t}$ number of achievements in the listener TIAV multimedia activities during $t$. Set is estimated at time personal characteristics of the listener denote $X_{\mathrm{C} J \mathrm{X}}^{t}=$ $\left(x_{m^{t}+p^{t}}^{t}, \ldots, x_{m^{t}+p^{t}+q^{t}}^{t}\right)$, where $q^{t}$ - number of evaluated characteristics of the listener during t. Thus, the assessment of competence listener is made based on a variety of grade $X^{t}=X_{\text {OКМ }}^{t} \cup X_{\text {MOP }}^{t} \cup X_{\text {СЛX }}^{t}=\left(x_{1}^{t}, \ldots, x_{r}^{t}, \ldots, x_{m^{t}+p^{t}+q^{t}}^{t}\right)$, with $x_{r}^{t} \in X_{r}^{t}$, where $X_{r}^{t}$ - the set of possible values of the variable $x_{r}^{t}$.

\section{Conclusions}

The theoretical significance of the results of the study is that the proposed methods, models, algorithms and software packages allow you to develop multimedia systems (instrumental complexes TIAV-multimedia systems) to process information resources combining text, sound, graphics, photos videos in one digital representation. Methods of construction of the control system of discrete-continuous processes processing of information resources in multimedia systems TIAV-derived software tools allow to reduce the complexity and significantly reduce the time of design and implementation of a control system of discrete-continuous processes of treatment of various types of information in TIAV-multimedia systems, for such important and resource-intensive tasks such as diagnostics of technical objects and systems management of complex organizational and production systems and processes, marketing and other monitoring. The practical significance of the results and lessons learned to develop static and dynamic TIAV-multimedia system not only significantly reduce the available hitherto gap between world and domestic levels in solving the problems of such theoretical importance and complexity, but also provide a methodological framework for the training of qualified personnel in the leading technical universities of Uzbekistan. TIAV-use multimedia systems provides human lightness perception information as the person is substantially different from the computer means and information processing means having a shape perception convenient for humans. Using TIAV-multimedia systems provides ease of perception provides an opportunity not only to be contemplative ready multimedia material, but also a party to its creation, transformation, operational use. Intensification TIAV-use multimedia systems is achieved through the creation of new ways of learning, ability to store, fill up, organize, and use promptly banks information on any area of knowledge, due to the higher than traditional teaching methods, the degree of clarity, features a wide replicate the best teachers reflected in its software products.

TIAV multimedia technology will make oral visibility from a static to a dynamic, i.e., it will be possible to monitor the processes studied in time. Simulate the processes that evolve over time; ability to interactively change the parameters of these processes - a very important didactic advantage of multimedia learning systems. Especially a lot of educational problems related to the fact that the demonstration of the phenomena being studied can't be held in the classroom, in the case of multimedia are the only possible today.

The program complex multimedia system based on network technologies support developed methodology is a unique, modern tools, including an interrelated set of means of building automation control system of discrete-continuous processes of treatment of various types of information in TIAV-multimedia systems, at all stages of the life cycle and provide project management to create a control system of discrete-continuous processes of information processing in TIAV-multimedia systems, as a whole, depending on the task, the set of available resources and the specific model of the life cycle, all of which is 
determined by the theoretical model developed multimedia technology.

\section{Acknowledgements}

The management of flow processes in the multimedia activities is an important scientific problem that requires self-study and includes an important element of the production of the methodological principles of management of flow processes.

\section{REFERENCES}

[1] V.V. Lipaev Standardization of software quality evaluation. -Supplement to the "Information Technology", 2001, № 4.

[2] R.A. Farkhutdinov Universities Russia must prepare management professionals competitiveness / / Standards and Quality, 1999, № 6 .

[3] S.S. Beknazarova Discrete-continuous treatment processes information resources in tiav- multimedia systems, LAP LAMBERT Academic Publishing Gmb H\&Co. KG, Saarbrucken, Germany, 2015, 56. 